Oslo University Hospital) vom 25. November bezweifelt, dass i.v. Injektionen wie etwa Epinephrin im Verlauf der Reanimation sinnvoll sind. Es wurden zwar kurzfristig positive Effekte gefunden, die sich aber langfristig nicht ausbezahlten, $d . h$. kein Überlebensvorteil. Trifft das auch Ihre Erfahrung?

Klein: Die Arbeit zeigt sehr schön, wo wir stehen. Bisher wurde bei den Pharmaka nur für Amiodaron bei Kammerflimmern ein halbwegs positives Ergebnis erzielt. Entscheidend aber sind die möglichst rasche Defibrillation und gute Basisreanimationsmaßnahmen. Alle anderen Maßnah- men sind leider nur im Einzelfall, nicht aber studienmäßig hilfreich.

ICardioVasc: Was hat bei Ihnen in diesem Jahr in der Resuscitation-Session den stärksten Eindruck hinterlassen? Klein: Eindeutig die bereits erwähnte DARTStudie. Sie ist eine wirkliche Meilensteinstudie. Schade nur, dass mit dem veralteten Reanimationsschema 15:2 gearbeitet wurde, aber die Studie lief über viele Jahre, sodass die revolutionären Leitlinien aus dem Jahr 2005 bei Studienbeginn noch nicht existierten.

Das Gespräch führte Jochen Aumiller. In

\title{
Wegweisende DART-Studie
}

DART steht für „Dispatcher Assisted Resuscitation Trial. Randomized trial of bystander CPR comparing chest compression alone versus chest compression plus ventilation“. Präsentator der Studie im Rahmen der Jahrestagung der American Heart Association in Orlando war Thomas D. Rea von der „Division of Emergency Medical Services, Public Health Seattle and King County (University of Washington)“.

Eine frühere Studie aus Seattle von Hallström hatte tendenziell bessere Ergebnisse bei alleiniger Kompression durch Laien bei vermutetem plötzlichem Herzstillstand ergeben, das Rettungsteam war in dieser Studie bereits sehr früh vor Ort.

\section{Alleinige Thoraxkompression führt zu besserem Überleben}

Daraus wurde nun die Studienhypothese von DART abgeleitet: Alleinige Thoraxkompression (CC) durch Laien führt zu besserem Überleben als zusätzliche Beatmung (Schema noch 15:2).

Drei Rettungsstellen nahmen Randomisierung nach Alarmierung vor. Neurologisches Outcome konnte nur in zwei Stellen gesichert werden. In die Studie aufgenommen wurden insgesamt 1941 Notfälle, davon 989 mit CC allein und 952 mit CC plus Ventilation. Im Mittel waren die Patienten 63 Jahre, bei etwa $70 \%$ lag eine kardiale Ätiologie vor, bei $43 \%$ ereignete sich der Notfall vor Zeugen, $87 \%$ der Patienten kollabierten zu Hause. Der Rettungsdienst war durchschnittlich in 6,5 min vor Ort, Defibrillatoren wurden bei $33 \%$ der Notfälle eingesetzt.

\section{Die Ergebnisse}

Die Überlebensquote (Krankenhausentlassung) betrug in der CC-Gruppe $12,8 \%$ und $10,7 \%$ in der Vergleichsgruppe CC plus Ventilation ( $p=$ $0,15)$. Das neurologische Outcome lag bei $14,8 \%$ in der CC-Gruppe und bei $11 \%$ beim kombinierten Vorgehen CC plus Ventilation ( $p=0,041$ ).

H. H. Klein, Idar-Oberstein I 